Lenice de Castro Mendes Villela'

Edna Maria Rezende ${ }^{\mathrm{I}}$

Eliane de Freitas Drumond"

Lenice Harumi Ishitani"

Gustavo Malta Ladeira

Carvalho"II

\section{Utilização da imprensa escrita na qualificação das causas externas de morte}

\section{Use of the printed press for qualification of external causes of death}

RESUMO

OBJETIVO: Complementar a informação sobre a causa básica de morte em óbitos por causas externas com uso de notícias veiculadas em jornais.

MÉTODOS: Estudo de investigação de 153 óbitos por causas externas ocorridas em residentes de Belo Horizonte, MG, em 2008. Construiu-se o banco de dados denominado imprensa, com informações de jornais de grande circulação nacional e estadual, o qual foi relacionado ao banco de dados do Sistema de Informações de Mortalidade. Os dados foram analisados nos programas EpiInfo e o Link-plus. A concordância dos resultados foi avaliada pelo coeficiente kappa.

RESULTADOS: Foram localizadas 1.530 notícias sobre acidentes e violências, 201 das quais foram pareadas às declarações de óbito do banco do Sistema de Informações de Mortalidade e 153 notícias eram de residentes de Belo Horizonte. As principais causas de morte identificadas nos bancos foram agressões e acidentes de transporte. No banco imprensa, agressões e acidentes de transporte corresponderam a $86,3 \%$, outros acidentes $7,8 \%$, eventos de intenção indeterminada 4,6\%, e casos de intervenção legal 1,3\%. Após a complementação com o banco imprensa houve aumento nos óbitos por acidentes de automóveis $(220,0 \%)$ e motos $(100,0 \%)$, o que resultou em diminuição do número de óbitos por causa indeterminada e por acidentes de transporte não especificados notificados no Sistema de Informações de Mortalidade.

CONCLUSÕES: Notícias de jornais têm grande potencial para qualificar e complementar a causa básica de morte em óbitos por causas externas no Sistema de Informações de Mortalidade, principalmente daqueles por acidentes de transporte.

DESCRITORES: Registros de Mortalidade. Causas Externas. Violência. Acidentes de Trânsito, mortalidade. Causa Básica de Morte. Sistemas de Informação. Imprensa.

\author{
Correspondência | Correspondence: \\ Lenice de Castro Mendes Villela \\ R. Marechal Bittencourt, 895 Ap 02 \\ Gutierrez \\ 30430-200 Belo Horizonte, MG, Brasil \\ E-mail: lenicevillela@gmail.com
}

Recebido: 10/6/2011

Aprovado: 16/2/2012

Artigo disponível em português e inglês em: www.scielo.br/rsp
Infantile de Sú Enfermagem Materno Enfermagem. Universidade Federal de Minas Gerais (UFMG). Belo Horizonte, MG Gerência de Vigilância em Saúde e

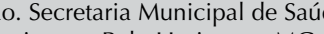
Delo Horizonte, MG

Curso de Graduação em Enfermagem. UFMG. Belo 


\begin{abstract}
OBJECTIVE: To supplement the information on the underlying cause of death due to external causes through using news carried in newspapers.

METHODS: This study investigated 153 deaths due to external causes among people living in Belo Horizonte, Southeastern Brazil, in 2008. A database named "press" was constructed using information from large-circulation national and state-level newspapers, and this was correlated with the database of the Brazilian mortality information system. The data were analyzed using the EpiInfo and Link-plus software. The concordance of the results was assessed using the kappa coefficient.
\end{abstract}

RESULTS: A total of 1,530 news items on accidents and violence were located, and 201 of these items were matched with death certificates in the mortality information system; 153 items referred to people living in Belo Horizonte. The main causes of death identified in the databases were aggression and traffic accidents. In the press database, aggression and traffic accidents accounted for $86.3 \%$, other accidents $7.8 \%$, events of undetermined intent $4.6 \%$ and legal intervention cases $1.3 \%$. After supplementation using the press database, there were increases in the numbers of deaths due to car accidents $(220.0 \%)$ and motorcycle accidents $(100.0 \%)$, which resulted in a decrease in the numbers of deaths due to indeterminate causes and unspecified traffic accidents notified in the mortality information system.

CONCLUSIONS: News in newspapers has great potential for qualifying and supplementing the information on the underlying cause of death due to external causes in the mortality information system, particularly regarding deaths due to traffic accidents.

DESCRIPTORES: Mortality Registries. External Causes. Violence. Accidents, Traffic, mortality. Underlying Cause of Death. Information Systems. Press.

\title{
INTRODUÇÃO
}

O impacto gerado pelos acidentes e violências na saúde das populações do Brasil e da maioria dos países é conhecido principalmente pela análise dos dados de mortalidade. ${ }^{10}$ As causas externas responderam por 13,6\% dos óbitos ocorridos em 2008 no Brasil, segundo dados da Rede Interagencial de Informações para a Saúde. ${ }^{a}$ Embora a interiorização da violência seja observada em todo o País, a mortalidade proporcional por causas externas continua em patamares mais elevados nas capitais do que nas cidades do interior. ${ }^{15}$

A magnitude e elevação dos coeficientes de agressões e acidentes de transporte em população cada vez mais jovem são marcantes no padrão epidemiológico brasileiro da mortalidade por causas externas. As taxas são extraordinariamente mais altas em adolescentes e adultos jovens, especialmente do sexo masculino dos 15 aos 29 anos, do que na população como um todo. ${ }^{15}$

A causa de morte é uma das mais importantes informações da declaração de óbito e fundamental para que seja conhecido o estado da saúde das populações. Nos casos de mortes violentas ou não naturais, a declaração do óbito, inclusive a causa mortis, deverá ser preenchida e fornecida pelo serviço médico legal, conforme determinado pelo Código Penal Brasileiro ${ }^{\mathrm{b}}$ e garantido na Resolução $n^{\circ}$ 1.601/00 do Conselho Federal de Medicina. ${ }^{c}$ Quando a causa da morte for uma lesão decorrente de causa externa, a circunstância relacionada ao evento mórbido deve ser mencionada e selecionada como causa básica da morte. Muitas vezes, o médico legista menciona apenas a natureza das lesões e não

a Rede Interagencial de informação para a Saúde. Indicadores básicos para a saúde no Brasil: conceitos e aplicações. Brasília: Organização Pan-Americana da Saúde; 2010.

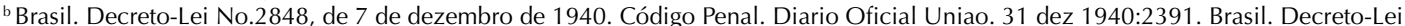
No.2848, de 7 de dezembro de 1940. Código Penal. Diario Oficial Uniao. 31 dez 1940:2391.

${ }^{c}$ Conselho Federal de Medicina (BR). Resolução n ${ }^{\circ} 1.641$. Veda a emissão, pelo médico, de Declaração de Óbito nos casos em que houve atuação de profissional não-médico e dá outras providências. Diario Oficial Uniao. 29 jul 2002;Seção 1:22. 
explicita a circunstância da morte. ${ }^{7}$ Para suprir essa lacuna, é realizada a busca ativa sistemática de informações sobre as circunstâncias das mortes por causas externas no Instituto de Medicina Legal (IML) de Belo Horizonte desde 2000. As fontes de informações utilizadas são os relatórios médicos de encaminhamento dos corpos, relatórios de outros profissionais, solicitações de perícias e laudos de perícia médica. Porém, essas fontes são insuficientes para a adequada qualificação das informações sobre as circunstâncias das mortes por causas externas, i.e., acidentes e violência. ${ }^{2,6,8,12,14} \mathrm{Em}$ decorrência disso, as mortes por eventos cuja intenção é indeterminada representaram proporção de $10,1 \%$ no município, ligeiramente superior à média brasileira de $8,7 \%$ em 2007. ${ }^{5}$ A qualidade das análises de dados sobre mortalidade fica comprometida quando proporção considerável de causas de morte é classificada como fatos ou eventos de causa indeterminada. ${ }^{11,12}$ Algumas regiões brasileiras utilizam notícias sobre acidentes e violências publicadas em jornais de grande circulação como fontes complementares da informação sobre as circunstâncias das mortes. ${ }^{5}$ Informações geradas por notícias veiculadas em jornais são rotineiramente comparadas às do Sistema de Informações de Mortalidade (SIM) no estado do Rio de Janeiro e, em alguns casos, incorporadas em seu banco de dados. ${ }^{9}$

O objetivo do presente trabalho foi complementar a informação sobre a causa do óbito por causas externas com uso de notícias veiculadas em jornais.

\section{MÉTODOS}

Estudo de investigação de óbitos por causas externas em jornais de grande circulação em Minas Gerais durante o ano de 2008.

A busca ativa de todos os casos de acidentes e violências, independentemente de ter resultado em morte, foi feita pela leitura diária de notícias veiculadas em três jornais estaduais ("Estado de Minas", "O Tempo", "Hoje em Dia") e um nacional ("Folha de S. Paulo"). Essas fontes foram escolhidas por sua grande circulação e a potencial cobertura dos fatos. Cada notícia selecionada foi notificada em um instrumento de coleta de dados: "ficha de notificação de urgência e emergência”, utilizado em inquérito sobre acidentes e violências em Belo Horizonte em 2007 padronizado pelo Ministério da Saúde. ${ }^{\mathrm{d}}$

Foram registrados: nome; idade; data da notificação; data e local de ocorrência (município, bairro, logradouro); intencionalidade (acidente, violência, ignorado); tipo de ocorrência; natureza da lesão. Os dados foram digitados em um banco denominado Imprensa, desenvolvido no EpiInfo 2000, versão 3.5.1.
As circunstâncias das mortes foram obtidas por busca ativa rotineira no IML quando necessário, seguida de sua codificação por técnicos treinados pelo Centro Brasileiro para Classificação de Doenças.

Realizou-se o relacionamento probabilístico (linkage) dos dados do banco imprensa com o banco do SIM a partir do software Link-Plus versão 2.0. As variáveis de relacionamento utilizadas foram: nome, idade, sexo e data do óbito. Seis grupos foram definidos para avaliar a concordância entre as causas de morte obtidas em cada fonte, utilizando os códigos de três dígitos da Classificação Estatística Internacional de Doenças e Problemas Relacionados à Saúde, Décima Revisão (CID-10): ${ }^{13}$ AGRES (agressão - X85 a Y09), ATRANSP (acidente de transporte - V01 a V99), AUTO (autoprovocada - X60 a X84); INTIND (intenção indeterminada - Y10 a Y34), OUTRO-AC (outros acidentes - W00 a X59) e LEGAL (intervenção legal - Y35 a Y36). Os pares que apresentaram 95\% de concordância entre as variáveis de pareamento foram considerados verdadeiros.

As notícias de jornal qualificaram as informações do SIM em caso de não concordância entre as causas de morte nos bancos, quando nelas havia melhor especificação sobre a circunstância do óbito, como: o local de ocorrência; o tipo de arma utilizada; o tipo de acidente (de trânsito, não de trânsito ou não especificado), o meio de transporte (pedestre, bicicleta, motocicleta, triciclo, automóvel, caminhonete, veículo de transporte pesado, ônibus, outros meios de transporte terrestre, transporte por água, transporte aéreo e espacial) e a qualidade da vítima (condutor ou passageiro).

Utilizou-se o programa Epidat 3.1 para calcular o coeficiente de concordância e se a alteração da causa básica após a investigação nos jornais foi significativa. O coeficiente estatístico kappa foi usado para avaliar a concordância entre os bancos. O kappa é uma medida de concordância interobservador e mede o grau de concordância além do que seria esperado pelo acaso. Tem como valor máximo 1, que apresenta concordância total e os valores próximos e abaixo de 0 , que indicam nenhuma concordância, ou a concordância foi exatamente a esperada pelo acaso, portanto discordância, mas seu valor não tem interpretação como intensidade de discordância. ${ }^{4}$

A pesquisa foi aprovada pelos Comitês de Ética em Pesquisa da Universidade Federal de Minas Gerais $\left(n^{\circ}\right.$ ETIC 638/08, em 17/12/2008) e da Secretaria Municipal de Saúde de Belo Horizonte ( ${ }^{\circ}$ CAAE 065.2008, em 18/12/2008).

d Ministério da Saúde (BR), Secretaria de Vigilância em Saúde, Departamento de Análise e Situação de Saúde. Viva: Vigilância de Violências e Acidentes, 2008 e 2009. Brasília: Ministério da Saúde; 2010. 


\section{RESULTADOS}

Ocorreram 1.853 óbitos por causas externas de residentes em Belo Horizonte em 2008 segundo dados do SIM (13,1\% do total de óbitos).

Foram identificados 1.530 acidentes e violências; $13,1 \%$ foram pareados às declarações de óbitos, dos quais 76,1\% ocorreram em Belo Horizonte. Os demais ocorreram em outros municípios mineiros ou em outros estados.

As agressões e os acidentes de transportes representaram 79,0\% dos óbitos no SIM (Figura 1). Os eventos de intenção indeterminada e os outros acidentes, como sufocação, esmagamento, afogamento, envenenamento, intoxicação, choque, entre outros, representaram o restante desses óbitos. Agressões e acidentes de transporte foram responsáveis por $86,3 \%$ dos óbitos no banco imprensa. Outros acidentes apresentaram maior número de óbitos $(7,8 \%)$ do que os eventos de intenção indeterminada (4,6\%). Destaca-se que no banco SIM nenhum óbito foi registrado como intervenção legal; no entanto, no banco imprensa $1,3 \%$ dos óbitos foram identificados por essa causa básica. Não houve pareamento de nenhum óbito autoprovocado (suicídios). O coeficiente kappa foi 0,80 , indicando concordância significativa entre todas as causas de morte violentas nos dois bancos.

Diferenças foram observadas após concordância dos agrupamentos por agressão e outros acidentes (Tabela 1). Dos 18 óbitos por intenção indeterminada no SIM, 12 tiveram qualificação da informação após investigação: metade migrou para agressões, cerca de $42,0 \%$ para acidentes de transporte e $8,0 \%$ para intervenção legal. Ressalta-se que, dos 18 óbitos, $33,3 \%$ permaneceram como eventos de intenção indeterminada.

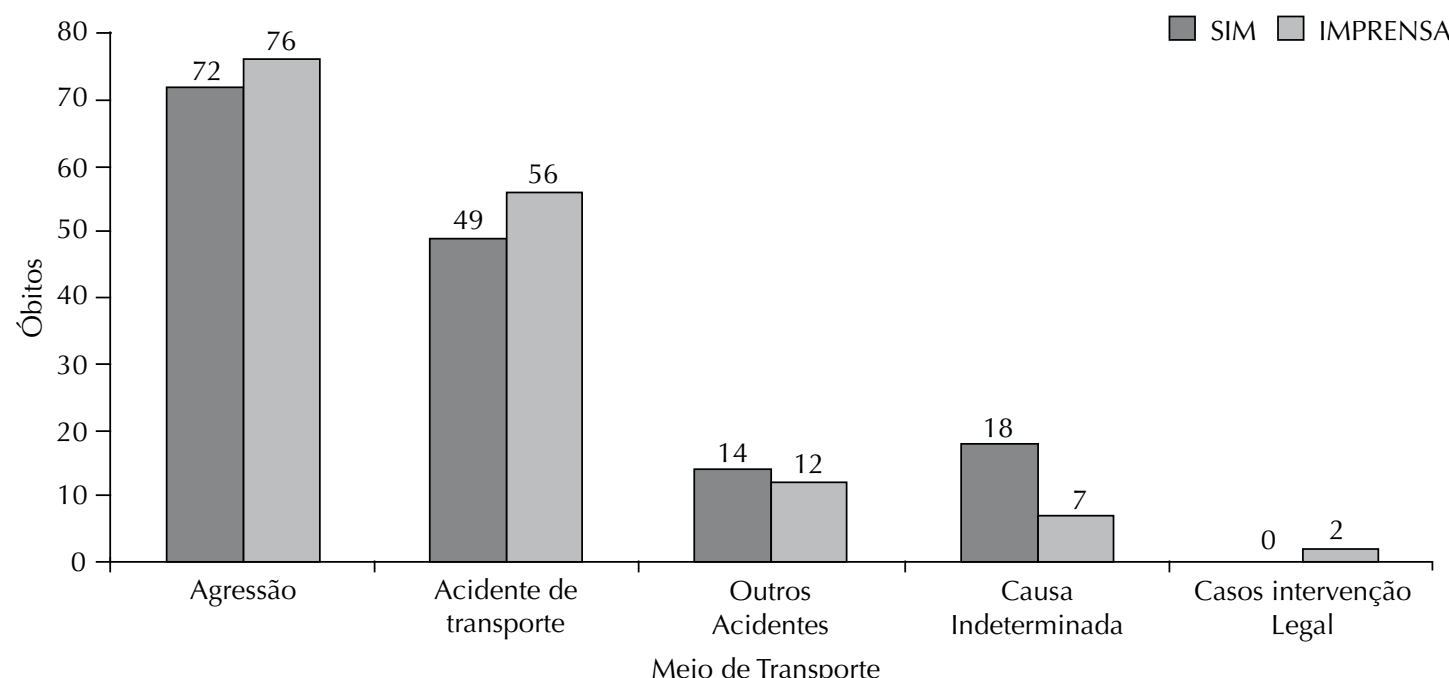

Fonte: Sistema de Informação de Mortalidade / Gerência de Epidemiologia e Informação / Secretaria Municipal de Saúde de Belo Horizonte - Minas Gerais

Figura 1. Principais grupos de causas externas de morte, Sistema de Informação de Mortalidade e imprensa. Belo Horizonte, MG, 2008.

Tabela 1: Principais grupos de causas externas de morte segundo Sistema de Informação de Mortalidade e imprensa. Belo Horizonte, MG, 2008.

\begin{tabular}{|c|c|c|c|c|c|c|}
\hline \multirow{2}{*}{$\begin{array}{l}\text { Sistema de Informação de } \\
\text { Mortalidade }\end{array}$} & \multicolumn{6}{|c|}{ Imprensa } \\
\hline & Agressão & $\begin{array}{l}\text { Acidente de } \\
\text { transporte }\end{array}$ & $\begin{array}{c}\text { Intenção } \\
\text { indeterminada }\end{array}$ & $\begin{array}{l}\text { Intervenção } \\
\text { legal }\end{array}$ & $\begin{array}{l}\text { Outros acidentes } \\
\text { de transporte }\end{array}$ & Total \\
\hline Agressão & 69 & 1 & 0 & 1 & 1 & 72 \\
\hline Acidente de transporte & 0 & 49 & 0 & 0 & 0 & 49 \\
\hline Intenção indeterminada & 6 & 5 & 6 & 1 & 0 & 18 \\
\hline Intervenção legal & 0 & 0 & 0 & 0 & 0 & 0 \\
\hline Outros acidentes de transporte & 1 & 1 & 1 & 0 & 11 & 14 \\
\hline Total & 76 & 56 & 7 & 2 & 12 & 153 \\
\hline
\end{tabular}

Fonte: Sistema de Informação de Mortalidade / Gerência de Epidemiologia e Informação / Secretaria Municipal de Saúde de Belo Horizonte - Minas Gerais 
Obteve-se concordância moderada entre os bancos $($ kappa $=0,41)$. Houve aumento de $220,0 \%$ nos óbitos envolvendo automóveis e de $100 \%$ nos acidentes com motocicleta e bicicleta (Figura 2). Os 16 óbitos atribuídos à pessoa traumatizada em acidente com um veículo não especificado (código V89.9) no SIM puderam ser identificados pelo banco imprensa: 11 casos relativos a automóvel, três a motocicletas e dois óbitos por atropelamento (Tabela 2).

\section{DISCUSSÃO}

Apesar da concordância significativa entre as informações dos bancos imprensa e SIM, a qualidade dos dados sobre causas externas de morte em Belo Horizonte precisa ser aprimorada, mesmo após a investigação rotineira dos óbitos no IML. Isso devido à elevada proporção de óbitos por eventos de intenção indeterminada e por acidentes de transporte não especificados que puderam ser qualificados pelas noticias de jornais. A busca ativa sistemática e rotineira de informações nos prontuários do IML mostrou-se insuficiente para a qualificação das circunstâncias da morte por acidente de transporte, especialmente por sua magnitude e evitabilidade. A caracterização do meio de transporte é um dos fatores mais importantes para políticas de monitoramento e de prevenção dos acidentes de transporte, assim como a especificação de pedestre, ciclista, motociclista, condutor ou passageiro. Há escassez de informações sobre o meio de transporte utilizado pela vítima nas fontes consultadas (boletins de ocorrência, encaminhamentos hospitalares, informações de familiares e outras). Conforme ressaltado por Baullinger et $\mathrm{al}^{1}$ (2001), embora com algumas limitações, a análise de notícias de jornais auxilia os profissionais na qualificação das informações sobre óbitos e pode ser usada como ferramenta complementar para a vigilância.

O SIM tem grande potencial como fonte de dados para análise visando à implantação e à avaliação de políticas públicas para a redução das mortes violentas. Esse sistema de informações apresenta grandes avanços na sua cobertura e fidedignidade dos dados em Belo Horizonte e em todo o Brasil. No entanto, deve-se reforçar a importância para o correto preenchimento dos encaminhamentos, dos prontuários e da declaração de óbitos aos profissionais que encaminham corpos ao IML, policiais e profissionais de saúde e aos legistas. ${ }^{5,6,9}$

Parte considerável da informação de acidentes ou violências veiculadas pela imprensa escrita não entrou no pareamento com o banco do SIM, uma vez que os jornais podem não identificar corretamente as vítimas: deixam de incluir nome completo, sexo, idade e logradouro do acidente, quem é o motorista ou passageiro e o tipo de veículo envolvido. Outra limitação relaciona-se com os óbitos autoprovocados, pois esses geralmente não são divulgados nos jornais por questões éticas, familiares e jurídicas, e por isso não foram qualificados no estudo. Algumas causas externas praticamente não são veiculadas ou são deixadas em segundo plano quando se destaca uma notícia ou evento de grande importância para o estado ou País. Isso contribui para possível perda quantitativa no cruzamento dos dados. Apesar disso, as interferências por perdas de dados atribuíveis ao não pareamento não impactaram nos resultados obtidos.

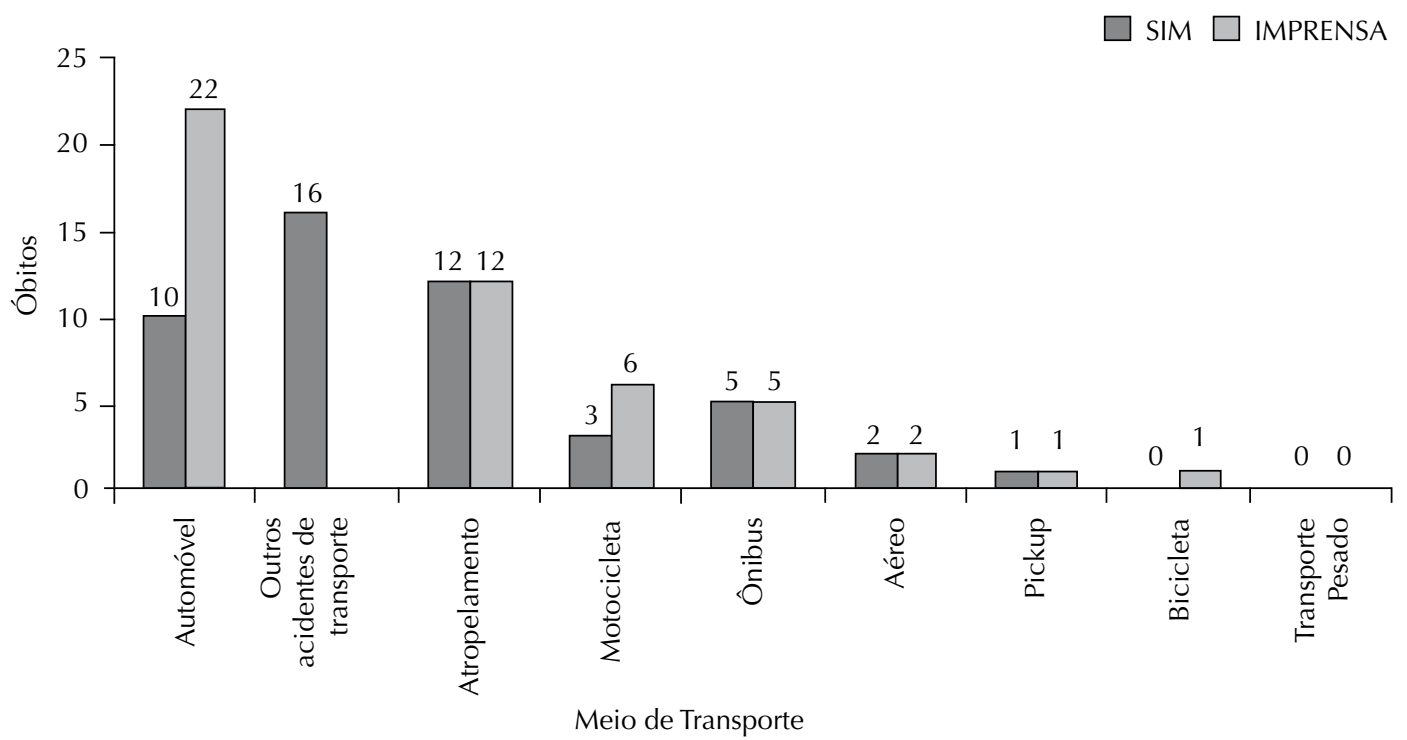

Fonte: Sistema de Informação de Mortalidade / Gerência de Epidemiologia e Informação / Secretaria Municipal de Saúde de Belo Horizonte - Minas Gerais

Figura 2: Óbitos segundo o meio de transporte, Sistema de Informação de Mortalidade e imprensa. Belo Horizonte, MG, 2008. 
Tabela 2. Óbitos segundo o meio de transporte, Sistema de Informação de Mortalidade e imprensa. Belo Horizonte, MG, 2008.

\begin{tabular}{|c|c|c|c|c|c|c|c|c|c|c|}
\hline \multirow{2}{*}{$\begin{array}{l}\text { Sistema de } \\
\text { Informação } \\
\text { de } \\
\text { Mortalidade }\end{array}$} & \multicolumn{10}{|c|}{ Imprensa } \\
\hline & Aéreo & Atropelamento & Automóvel & Bicicleta & Motocicleta & Ônibus & $\begin{array}{c}\text { Transporte } \\
\text { pesado }\end{array}$ & Pickup & $\begin{array}{c}\text { Outros } \\
\text { acidentes } \\
\text { transporte }\end{array}$ & Total \\
\hline Aéreo & 2 & 0 & 0 & 0 & 0 & 0 & 0 & 0 & 0 & 2 \\
\hline Atropelamento & 0 & 9 & 1 & 1 & 1 & 0 & 0 & 0 & 0 & 12 \\
\hline Automóvel & 0 & 1 & 9 & 0 & 0 & 0 & 0 & 0 & 0 & 10 \\
\hline Bicicleta & 0 & 0 & 0 & 0 & 0 & 0 & 0 & 0 & 0 & 0 \\
\hline Motocicleta & 0 & 0 & 1 & 0 & 2 & 0 & 0 & 0 & 0 & 3 \\
\hline Ônibus & 0 & 0 & 0 & 0 & 0 & 5 & 0 & 0 & 0 & 5 \\
\hline $\begin{array}{l}\text { Transporte } \\
\text { pesado }\end{array}$ & 0 & 0 & 0 & 0 & 0 & 0 & 0 & 0 & 0 & 0 \\
\hline Pickup & 0 & 0 & 0 & 0 & 0 & 0 & 0 & 1 & 0 & 1 \\
\hline $\begin{array}{l}\text { Outros } \\
\text { acidentes } \\
\text { transporte }\end{array}$ & 0 & 2 & 11 & 0 & 3 & 0 & 0 & 0 & 0 & 16 \\
\hline Total & 2 & 12 & 22 & 1 & 6 & 5 & 0 & 1 & 0 & 49 \\
\hline
\end{tabular}

Fonte: Sistema de Informação de Mortalidade / Gerência de Epidemiologia e Informação / Secretaria Municipal de Saúde de Belo Horizonte - Minas Gerais

Segundo Kucinski, ${ }^{3}$ (2000) a utilização da imprensa como fonte de investigação pode ser questionada. Sendo um meio de disseminação de informações para ampliar o conhecimento da população sobre determinado fato ou evento, a mídia pode ser tendenciosa e especulativa, supervalorizando aspectos ainda não comprovados ou esclarecidos. Souza et al ${ }^{16}$ (2006) ressaltam que, embora a imprensa escrita utilize informações de forma acrítica e sensacionalista, é um recurso alternativo para complementar e enriquecer os dados sobre a mortalidade por violência e acidentes, principalmente de um sistema de informação com problemas sobre sua qualidade.

A construção de um banco de dados a partir da imprensa escrita acrescentou importantes informações àquelas registradas no SIM, contribuindo para complementar e qualificar a causa básica de óbito por causas externas, especialmente nos eventos de intenção indeterminada e por outros acidentes de transporte não especificados.

O elevado número de notícias de jornais sobre acidentes e violências registrados neste estudo valoriza essa fonte de informação. Torna-se necessário avaliar a qualidade das notícias veiculadas na imprensa escrita, a capacidade de difundir informações fidedignas e sua potencialidade para sinalizar ações preventivas na redução dos acidentes e violências. As notícias devem ser acrescidas às políticas públicas de transporte urbano e segurança pública no enfrentamento da violência nos grandes centros urbanos.

A utilização rotineira da vigilância de notícias sobre causas externas veiculadas em jornais é importante para comparação e incorporação das informações no SIM. Articulações entre os serviços de saúde e a mídia poderiam fortalecer essa iniciativa. 


\section{REFERÊNCIAS}

1. Baullinger J, Quan L, Bennett E, Cummings P, Williams K. Use of Washington State newspapers for submersion injury surveillance. Inj Prev. 2001;7(4):339-42. DOI:10.1136/ip.7.4.339

2. Drummond Jr M, Lira MTA, Freitas M, Nitrini TMV, Shibao K. Avaliação da qualidade das informações de mortalidade por acidentes não especificados e eventos com intenção indeterminada. Rev Saude Publica. 1999;33(3):273-80.

DOI:10.1590/S0034-89101999000300008

3. Kucinski B. Jornalismo, saúde e cidadania. Interface (Botucatu). 2000;4(6):181-6. DOI:10.1590/S1414-32832000000100025

4. Landis JR, Koch GG. The measurement of observer agreement for categorical data. Biometrics. 1977;33(1):159-74. DOI:10.2307/2529310

5. Laurenti R, Mello Jorge MHP, Gotlieb SLD. Informação em mortalidade: o uso das regras internacionais para a seleção da causa básica. Rev Bras Epidemiol. 2009;12(2):195-203.

DOI:10.1590/\$1415-790X2009000200009

6. Laurenti R, Mello Jorge MHP, Gotlieb SLD. O Sistema de Informações sobre Mortalidade: passado, presente e futuro. São Paulo: Centro Brasileiro de Classificação de Doenças; 2006. (Série Divulgação, 11).

7. Lozada EMK, Mathias TAF, Andrade SM, Aidar T. Informações sobre mortalidade por causas externas e eventos de intenção indeterminada, Paraná, Brasil, 1979 a 2005. Cad Saude Publica. 2009;25(1):223-8. DOI:10.1590/S0102-311X2009000100024

8. Matos SG, Proieitti FA, Barata RCB. Confiabilidade da informação sobre mortalidade por violência em Belo
Horizonte, MG. Rev Saude Publica. 2007;41(1):76-84. DOI:10.1590/S0034-89102007000100011

9. Mello Jorge MHP, Cascão AM, Silva RC. Acidentes e violências: um guia para o aprimoramento da qualidade de sua informação. São Paulo: Centro da OMS para a Classificação de Doenças em Português; 2003. (Série Divulgação, 10).

10. Mello Jorge MHP, Gawryszewki VP, Latorre MRDO. Análise dos dados de mortalidade. Rev Saude Publica. 1997;31(Supl 4):5-25.

DOI:10.1590/S0034-89101997000500002

11. Mello Jorge MHP, Laurenti R, Gotlieb SLD. Análise da qualidade das estatísticas vitais brasileiras: a experiência da implantação do SIM e do SINASC. Cienc Saude Coletiva. 2007;12(3):643-54. DOI:10.1590/S1413-81232007000300014

12. Mello Jorge MHP, Laurenti R, Di Nubila HBV. O óbito e sua investigação: reflexões sobre alguns aspectos relevantes. Rev Bras Epidemiol. 2010;13(4):561-76. DOI:10.1590/S1415-790X2010000400002

13. Organização Mundial da Saúde. CID-10/ Organização Mundial da Saúde. 2 ed. São Paulo: Edusp; 1994.

14. Santo AH. Causas mal definidas de morte e óbitos sem assistência. Rev Assoc Med Bras. 2008;54(1):23-8. DOI:10.1590/S0104-42302008000100016

15. Souza ER, Lima MLC. Panorama da violência urbana no Brasil e suas capitais. Cienc Saude Coletiva. 2006;11(Supl):1211-22. DOI:10.1590/S1413-81232006000500011

16. Souza NSS, Portinho BG, Barreiros MF. Acidentes de trabalho com óbito registrados em jornais no estado da Bahia. Rev Baiana Saude Publica. 2006;30(1):77-89.

Carvalho GML foi apoiado pela Fundação de Amparo à Pesquisa do Estado de Minas Gerais (FAPEMIG - Edital Único 01/2010; bolsa de iniciação científica).

Os autores declaram não haver conflitos de interesse. 